\title{
Assessment of the ITER high-frequency magnetic diagnostic set
}

\author{
D. Testa ${ }^{\mathrm{a}, *}$, H. Carfantan ${ }^{\mathrm{b}}$, M. Toussaint ${ }^{\mathrm{a}}$, R. Chavan ${ }^{\mathrm{a}}$, Y. Fournier ${ }^{\mathrm{c}}$, J. Guterl $^{\mathrm{a}}$, J.B. Lister ${ }^{\mathrm{a}}$, T. Maeder $^{\mathrm{c}}$, \\ J.-M. Moret ${ }^{\mathrm{a}}$, A. Perez ${ }^{\mathrm{a}}$, F. Sanchez ${ }^{\mathrm{a}}$, B. Schaller ${ }^{\mathrm{a}}$, C. Slater $^{\mathrm{c}}$, M. Stoeck ${ }^{\mathrm{c}}$, G. Tonetti ${ }^{\mathrm{a}}$ \\ a Ecole Polytechnique Fédérale de Lausanne, Centre de Recherches en Physique des Plasmas, Lausanne, CH, Switzerland \\ ${ }^{\mathrm{b}}$ Laboratoire d'Astrophysique de Toulouse - Tarbes, Université de Toulouse, CNRS, FR, Switzerland \\ ' Laboratoire de Production Microtechnique (LPM), Ecole Polytechnique Fédérale de Lausanne, CH, Switzerland
}

\section{A R T I C L E I N F O}

Article history:

Available online 7 May 2011

\section{Keywords:}

ITER

High-frequency magnetic diagnostic

\begin{abstract}
A B S T R A C T
The ITER high-frequency (HF) magnetic diagnostic system has to provide essential measurements of MHD instabilities with $\left|\delta B_{\mathrm{MEAS}} / B_{\mathrm{POL}}\right| \sim 10^{-4}(\sim 1 \mathrm{G})$ for frequencies up to $2 \mathrm{MHz}$ to resolve toroidal mode numbers $(n)$ in the range $|n|=10$ to $|n|=50$. A review of the measurement requirements for HF MHD instabilities in ITER was initiated during the TW4 work-program and led to significant interest for physics and real-time control issues in measuring modes with $\left|\delta B_{\mathrm{MEAS}}\right|$ as low as $\sim 10^{-3} \mathrm{G}$ at the position of the sensors, with $|n| \leq 30$ and poloidal mode numbers $|m| \sim 2|n|$ up to $|n| \sim 15$, for a frequency range extending up to $\sim 500 \mathrm{kHz}$. We have examined the ability of the current ITER design for the individual sensors and the diagnostic system as a whole to meet these needs, and have explored what adjustments to the design (of the individual sensors and/or of the system as a whole) or to the requirements would be needed to meet them when considering different hypothesis for the financial costs and risk management over the ITER life-time. First, we find that the proposed diagnostic layout, with 168 sensors in total, does not meet the more stringent measurement requirements and risk management criteria: these can only be met by a revision of the design requiring 350-500 sensors, depending on different costing and risk management options. Second, we find that the current design for the ITER HF Mirnov-type pick-up coil could be usefully revised.
\end{abstract}

(C) 2011 Published by Elsevier B.V.

\section{Introduction}

The high frequency (HF) magnetic sensor for ITER is currently intended to be a conventional, Mirnov-type, pick-up coil, i.e. a wound wire on a ceramic insulating former. The nominal engineering specifications for this sensor and the measurement requirements for the HF magnetic diagnostic system are given in various ITER documents [1]. Collating information from them, these specifications call for a sensor with an effective area $0.03<(\mathrm{NA})_{\mathrm{EFF}}$ $\left[\mathrm{m}^{2}\right]<0.1$, to measure magnetic instabilities with amplitude in the range $10^{-4}<\left|\delta B_{\mathrm{MEAS}} / B_{\mathrm{POL}}\right|<10^{-2}$, and for frequencies in the range between $10 \mathrm{kHz}$ and $2 \mathrm{MHz}$; finally, this diagnostic system should have the capability of recognizing toroidal mode numbers $(n)$ up to $|n|<50$ in all ITER main operating scenarios. The HF magnetic sensors are foreseen to be installed primarily within a cut out in the back face of some of the blanket modules (with approximate dimension $50 \mathrm{~mm} \times 50 \mathrm{~mm} \times 50 \mathrm{~mm}$ ). Additional sensors can also be located along the horizontal and vertical edges of selected equatorial ports, with initial provisions for this having been already incorporated in the current design of these ports.

\footnotetext{
* Corresponding author. Tel.: +41 216933568.

E-mail address: duccio.testa@epfl.ch (D. Testa).
}

A wealth of literature exists describing HF magnetic diagnostic systems in existing fusion experiments, and the desirable requirements for the measurement of HF instabilities in present and future burning-plasma devices [2]. Summarizing from the available documentation, the target design parameters (effective area, electrical properties) for HF magnetic sensors in ITER should be such that this diagnostic system will be able to provide measurements of MHD instabilities in the frequency range between $10 \mathrm{kHz}$ and up to $\sim 500 \mathrm{kHz}$ (but not necessarily above $1 \mathrm{MHz}$ ), with magnitude as low as practically possible, in the range $\left|\delta B_{\mathrm{MEAS}} / B_{\mathrm{POL}}\right| \sim 10^{-6}$ or lower. This value is well below the ITER requirement of $\left|\delta B_{\text {MEAS }} / B_{\text {POL }}\right| \sim 10^{-4}$, as the latter is of similar order to the threshold in mode amplitude that is expected to cause stochastic fast ion transport [3]. Note also that values of $\left|\delta B_{\text {MEAS }}\right| \sim \mathrm{mG}$ are routinely measured by HF pick-up coils in all major tokamaks such as JET [4], ASDEX-U [5], MAST [6], DIII-D [7], JT60U [8], i.e. for modes which are far away from the stochasticity threshold. An effective area in the range $(\mathrm{NA})_{\mathrm{EFF}} \approx(0.05-0.10) \mathrm{m}^{2}$, as in the ITER requirement, is deemed to be sufficient to achieve these measurements. Finally, and on the basis of current usage on existing tokamaks, this diagnostic system shall have the capability of recognizing toroidal mode numbers up to $|n|=30$ (hence below the $|n|=50$ requirement) and poloidal mode numbers ( $m$ ) up to $|m| \sim 2|n|$ for $|n|<15$, i.e. assuming a resonant $q$-surface $q=m / n=2$, consistently with the 
ITER requirements for measuring low- $m /$ low- $n$ neoclassical tearing modes [9].

\section{Analysis of the measurement performance of the ITER HF magnetic diagnostic}

We have performed the baseline analysis and optimization of the ITER HF magnetic diagnostic system using a new approach, which revolves around the "sparse representation of the signal spectrum", as implemented in the SparSpec code [10]. Applied to the problem of integer mode number analysis, the main idea of SparSpec is to model the data as a large number of pure modes $k \in\left\{-F_{\mathrm{MAX}}, \ldots,+F_{\mathrm{MAX}}\right\}$, where $F_{\mathrm{MAX}}$ is much larger (typically a factor 2-3) than the largest integer mode number that can conceivably be present in the input dataset. Among the many representations fitting the data, we seek the one with the lowest number of nonzero amplitudes, i.e. the so-called sparse solution. Using SparSpec the mode-fitting solution is computed as the minimizer of the $\mathrm{L}_{1}$ penalized least-square criterion:

$J(x)=\frac{1}{2}\|\mathbf{y}-W \mathbf{x}\|^{2}+\lambda \sum_{k=-K}^{K}\left(\left|x_{k}\right|\right)$, with $\lambda \leq \lambda_{\text {MAX }}=\max \left(\left|W^{\mathrm{H}} \cdot y\right|\right)$

here $\mathbf{y}=\left\{y_{1}, y_{2}, \ldots, y_{\mathrm{N}}\right\}^{T}$ is the vector of data taken at position $p_{n}, W$ is an $N \times\left(2 F_{\mathrm{MAX}}+1\right)$ matrix with elements $\boldsymbol{W}_{n, k}=\exp \left(\mathrm{i} 2 \pi k p_{n}\right)$, for $n=\{1, \ldots, N\} ; \mathbf{x}$ is the vector of complex amplitudes $x_{k}$ associated with modes $k$ and $\boldsymbol{W}^{\mathrm{H}}$ is the Hermitian transposition of $\boldsymbol{W}$. The parameter $\lambda$ defines $\mathrm{L}_{1}$-penalization term for adding unnecessary solutions to the mode decomposition algorithm, which is related to the noise variance $\sigma^{2}$ in the measurements.

The Sparse Spectrum algorithm has been applied to a model dataset for various implementations of the ITER HF magnetic sensor geometry for $n$ - and $m$-number detection. The ITER measurement requirements and the expected measurements' errors and tolerances are explicitly considered in this algorithm to define the correct and the wrong detection of the modes. For the purpose of assessing the measurement performance of any given arrangement of HF magnetic sensors in ITER, we have considered that the acceptable error is $15 \%$ on the mode amplitude and \pm 0 on the mode number for low- $n(m)$ HF modes which are of importance for plasma protection and control and for real-time measurements. Conversely, a measurement error ranging from \pm 1 to \pm 3 is deemed to be acceptable for modes which are only of "physics relevance", for which the amplitude only needs to be measured within $\pm 30 \%$. As the ITER vacuum vessel is still undergoing design changes, a system optimization that takes fully into account in-vessel engineering constraints is not yet possible. Hence, in addition to the physics constraints for the measurement requirements, a "cost function" has been included in the optimization algorithm to reflect the currently foreseen procurement and installation costs for the sensors. This cost function is constructed as follows, on the basis of the analysis performed during preparation of the Project Plan for the ITER HF magnetic diagnostic system (also providing the ranges in the cost function given below):

(1) each individual sensors costs from 7 to 10 cost-units endto-end, i.e. from the initial $R \& D$, to the detailed design and manufacturing, and from installation to the final data acquisition;

(2) each high-resolution sensor in any of the equatorial ports bears an additional installation cost of 1-2 cost-units due to the different needs for mechanical fixing, requiring further R\&D work and additional mechanical interfaces with the vessel structure;

(3) each poloidal sensor located in the regions $60<\theta(\mathrm{deg})<120$ and $270<\theta(\mathrm{deg})<315$ bears an additional installation cost of $1-2$ cost-units, due to more difficult cabling access;
(4) each high-field side poloidal sensor located in the region $120<\theta(\mathrm{deg})<220$ bears an additional installation cost of $2-3$ cost-units, again due an even more difficult cabling access;

(5) each high-field side poloidal sensor located in the divertor region $220<\theta(\mathrm{deg})<270$ bears an additional installation cost of 4-7 cost-units, again due to an even more difficult in-vessel cabling access and to need for improved RF screening of image and eddy currents;

(6) if we have more than 8 toroidal sensors (including highresolution ones) in any one of the 9 machine sectors, the cost increases by 1-2 cost-units for each additional group of 8 sensors due to the need of installing one further cabling loom in that sector.

The ratio between the confidence level in the measurement performance, and the costs necessary to achieve this performance, as defined above, can then give an additional indication of the overall system performance, one where we have integrated physics and budgetary requirements: the highest ratio defines the cheapest (financially) way to obtain the satisfactory (and desired and/or required) measurement performance.

Four different tests have been considered to assess the measurement performance of the ITER HF magnetic diagnostic system [11]. First, we consider an input data set made only of white Gaussian noise of known variance $\sigma^{2}$, and we determine the $95 \%$ confidence level for not detecting any true mode. This allows us to assess if one particular sensor arrangement is more prone than the others to mistakenly recognize white noise as being high- $n(m)$ modes. Second, we consider the statistic of recognizing correctly the given input "real modes", to which white Gaussian noise of known $\sigma^{2}$ is added, vs. the occurrence of "false alarms", i.e. modes being detected which are not in the input dataset. The results of these simulations are very simple to interpret: when changing the input spectrum, the sensor arrangements giving the higher number of correctly detected modes and the lower values of false alarms, represent the best choice for installation. Third, we consider the resilience of the selected geometry against the loss of sensors through faults, taken to be between $10 \%$ and $30 \%$ on the basis of current usage on existing machines. A measure for this is given by the relative error on the fitting of the input spectrum for the cases of "all vs. not-all" sensors being used. Once the fitting error using all sensors is accepted as giving good measurements, the lower the relative error over the range of permutations of faulty sensors and variations in the input spectrum, the more robust is that geometry against the loss of sensors. Fourth, we consider the position of each individual sensor as not absolutely fixed, but that there is a given volume where the sensor has to be located. This adds a new free parameter, i.e. a tolerance on the nominal position of each sensor as given by in-vessel surveys, to which we have added the calibration errors and the uncertainties in the equilibrium reconstruction, giving an "effective tolerance" on the position of each sensor of $\pm 3 \mathrm{deg}$. This value of $\pm 3 \mathrm{deg}$ is much larger than the equivalent "angular span" of the coils casing, which is of the order of $<0.1 \mathrm{deg}$, and is based on our operational experience on the JET and TCV HF magnetic diagnostic systems. Given an input spectrum to be detected, we can then artificially move the initial position of each sensor within this \pm 3 deg spatial tolerance to achieve the "best" measurement performance, which has then no consequence for the in-vessel installation. Only when the optimization algorithm suggests a larger displacement of the sensors, we would then have to change their actual in-vessel position. Hence, the more the sensors are displaced to optimize the measurement performance, the less robust is the initial non-optimized geometry against variation in the input modes' spectra.

A first example of these analyses is shown in Fig. 1, which presents the confidence level for noise rejection ( $=100$ for complete rejection) for evenly and un-evenly (randomly: generated 
HF magnetic diagnostic system for ITER:

confidence level normalized to R\&D, procurement \& installation costs

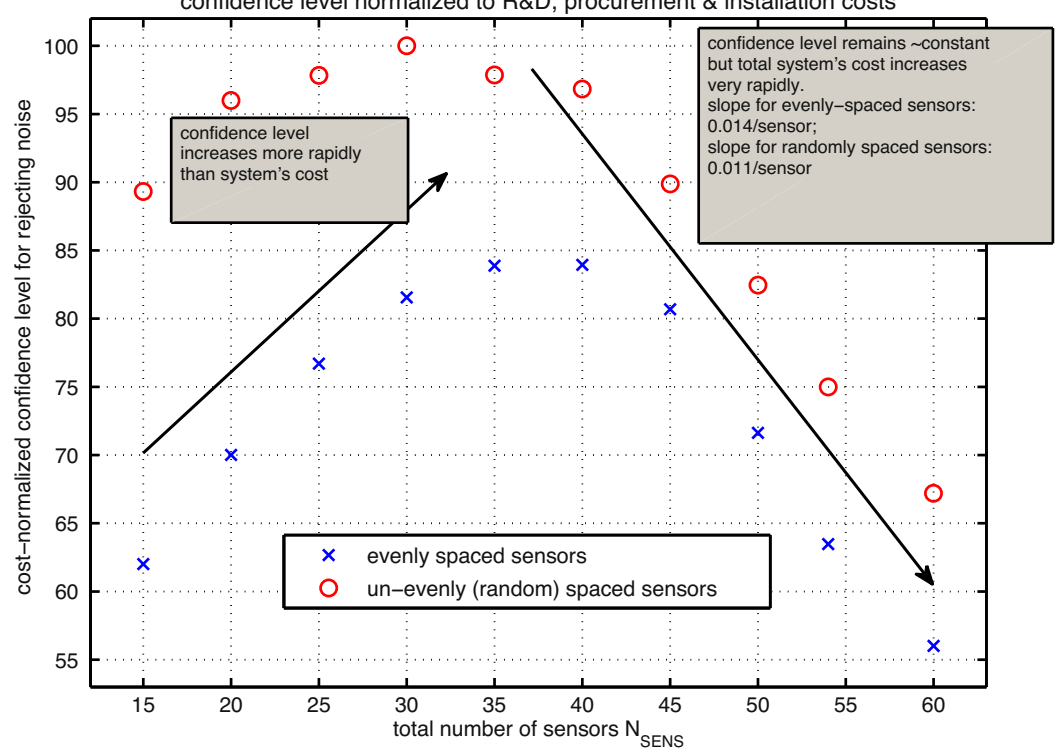

Fig. 1. The 95\% confidence level for noise rejection for evenly and randomly spaced sensors; this characteristic bell shape is obtained because above an optimal number of sensors the cost increases more rapidly than the confidence level for noise rejection, which remains almost constant.

using the Matlab function rand.m) spaced sensors, as function of the total number of sensors $N_{\text {SENS, }}$ and normalized with respect to the cost function for R\&D, procurement and installation. Note that an un-evenly spaced sensor distribution does not suffer from the well-known Nyquist limitation on the maximum measurable mode number that applies to evenly spaced sensor distributions, MaxMode $=N_{\text {SENS }} / 2$.

These results have been averaged for $\sigma=[0.0 \rightarrow 0.3]$, using different sensors distributions with the same $N_{\text {SENS }}$ (including toroidal-only sensors all at the same poloidal position, and poloidalonly sensors all at the same toroidal position), the results for all these distributions having been weighted over their individual cost function to account correctly for this value. We note immediately not only a local maximum in the normalized confidence level, but also the different behavior as the number of sensors increases. First, the best performing geometry has $\sim 30$ un-evenly spaced sensors, but needs around $\sim 40$ equi-spaced sensors. For an even higher number of sensors, small measurements errors starts to dominate over the reduced sensor spacing, hence the confidence level for noise rejection remains almost constant, so that false detection of high- $n(m)$ modes due to white noise cannot be avoided, but the cost function increases very rapidly, i.e. the measurement performance of the system becomes much less cost-efficient. Second, the reduction in the cost-normalized confidence level for noise rejection is sharper for an equi-spaced geometry as the number of sensors increases above its optimum value. This analysis leads to the conclusion that geometries made with sub-assemblies with spatial periodicities are inherently more prone than un-evenly distributed sensors to incorrect detection of high- $n(m)$ modes.

A second example of these analyses is shown in Table 1, which presents the results of the "false alarms" tests for the ITER nominal and a randomly spaced geometry, both with $\mathrm{NN}=16$ sensors, the latter having been optimized for measuring poloidal mode numbers up to $F_{\mathrm{MAX}}=30$. These results are again normalized with respect to the cost function for procurement and installation, and are averaged over a large number of simulations, run scanning $\sigma=0.0 \rightarrow 0.3$ and using from 2 to 5 input modes with 35,000 realizations for each individual mode with random relative amplitude, phase and mode number. Not only the number of false alarms is lower for the randomly spaced (optimized) geometry, but it also reaches a
Table 1

Summary results for the statistical analysis of false alarms for $m$-numbers for the same number of sensors NN, comparing the current ITER system design with the randomly spaced geometry, adding one high-resolution array in the equatorial port with NHR sensors. The optimal geometry is the one that minimizes the number of false alarms for $F_{\mathrm{MAX}}=30$.

\begin{tabular}{ccccc}
\hline NN & NHR & $F_{\text {MAX }}$ & $\begin{array}{l}\text { False alarms for } \\
\text { ITER geometry } \\
{[\%]}\end{array}$ & $\begin{array}{l}\text { False alarms for } \\
\text { optimal } \\
\text { geometry [\%] }\end{array}$ \\
\hline 16 & 0 & 20 & $15.3 \%$ & $7.3 \%$ \\
16 & 0 & 30 & $25.4 \%$ & $13.5 \%$ \\
16 & 0 & 45 & $43.7 \%$ & $15.2 \%$ \\
16 & 0 & 60 & $75.4 \%$ & $18.3 \%$ \\
16 & 3 & 30 & $8.4 \%$ & $4.7 \%$ \\
16 & 3 & 60 & $46.7 \%$ & $8.3 \%$ \\
16 & 7 & 30 & $4.3 \%$ & $2.8 \%$ \\
16 & 7 & 60 & $37.4 \%$ & $3.5 \%$ \\
16 & 10 & 30 & $7.9 \%$ & $2.4 \%$ \\
16 & 10 & 60 & $53.4 \%$ & $3.6 \%$ \\
16 & 15 & 30 & $18.4 \%$ & $9.6 \%$ \\
16 & 15 & 60 & $79.4 \%$ & $22.3 \%$ \\
\hline
\end{tabular}

local minimum for a lower number of sensors. The addition of highresolution arrays clearly improves the measurement performance of the nominal ITER geometry, but not up to the level of the randomly spaced optimized geometry. Conversely, we find that adding high-resolution sensors to a randomly distributed geometry, optimized without them, only improves the measurement performance up to a certain number of such high resolution sensors.

Finally, it is important to integrate the physics requirements for the HF magnetic diagnostic system with the guidelines given in the ITER risk management plan [12]. Considering the Risk Assessment Matrix developed for ITER, which combines the likelihood of occurrence for the problem with its consequences, and applying this scheme to the specific case of the HF magnetic diagnostic system, one can consider that the main risks to the measurement performance for HF MHD instabilities are due to (a) the loss of sensors over the ITER lifetime, and (b) unknown physics elements associated with a burning plasma that have not yet been explored in current devices nor predicted theoretically. As no backup measurements are foreseen for the high-frequency MHD instabilities, these risks can clearly be defined as having the so-called 
"high-impact". The obvious mitigation strategy for risk (a) is therefore that of implementing a sufficient redundancy in the total number of mechanically strong sensors, so that it can reasonably be assumed that enough sensors will survive until the last day of ITER operation. For mitigating risk (b), we need to implement the most flexible sensor geometries, so as to have the less limited detection capabilities for $n$ - and $m$-numbers, and those that are less dependent on continuing to have the same total number of sensors. Taking into account all these considerations, an optimized outline design for the ITER HF magnetic diagnostic system for toroidal and poloidal mode numbers analysis is proposed so as to have:

(a) toroidal mode numbers (main measurement arrays): on the low-field side, 2 arrays on the horizontal side of the equatorial ports, each array with 20-25 un-evenly spaced sensors plus $6 \times 5$ high resolution arrays located in each one of the equatorial ports used by the poloidal HF magnetic sensor system;

(b) toroidal mode numbers (for anti-ballooning mode analysis, redundancy and backup via diversity of location): on both the low- and high-field side, 2 arrays of 25-35 un-evenly spaced sensors located between $45 \mathrm{~cm}$ and $70 \mathrm{~cm}$ above and below the center of each equatorial port.

(c) poloidal mode numbers: one array of 20-30 un-evenly spaced and 5-7 high resolution sensors in 6 ports in non equi-distant machine sectors, not covering the divertor region and the areas around the top of the vessel, i.e. within $75<|\theta|(\mathrm{deg})<90$.

These geometries give a large redundancy in the toroidal and poloidal mode number measurements, and include in total $\sim 350$ to $\sim 500$ sensors for analysis of HF magneto-hydrodynamic instabilities in ITER. This is at least twice the number of $\sim 170 \mathrm{HF}$ magnetic sensors currently foreseen for ITER. With the implementation of these arrangements, we expect that the guidelines given in the ITER risk management plan will be fully satisfied.

\section{Assessment of the nominal ITER design for the HF magnetic sensor}

A coupled electro-magnetic, structural and thermal analysis was performed on the current Mirnov-type design for the HF sensor to find which of its components might be more susceptible to mechanical failure due to electromagnetic and/or thermal loads or fatigue [13]. We found as the main concern for the mechanical integrity of the sensor that differences in the thermal expansion of its various parts produce stress in the wire. Depending on the wire initial preload, this can break the wire or the ceramic supports, either directly or through mechanical fatigue, this considering very optimistic assumptions about the thermal heating and cooling of the pick-up coil assembly inside the blanket modules. This has therefore provided the essential focus for our prototyping program, where the manufacturing and mechanical characteristics of the prototypes were analysed with particular attention to the assembly process for the winding pack, by using three different types of guiding grooves on the ceramic support and two different materials for the wire itself.

We used the Rapid Prototyping technology to make the insulating former in Polyamide PA12 instead of the ceramic material envisaged in the ITER original design for the HF pick-up coil. The main purpose of using a Polyamide body is to allow winding of wires of different material to check the feasibility of this process on thin spacers, as this was the sole issue for the integrity of the ITER-designed Mirnov-type HF magnetic sensor in our FEM analysis. The different mechanical properties of Polyamide and ceramic do not affect the assessment of the winding process. Three different grooving designs were tested using a tungsten and a copper wire, and none was found suitable due to the likelihood of breakages of the thin edges in the spacers [13]. On the other hand, it was found that the electrical requirements (effective area, self-resonance frequency, self-inductance) could all be met concurrently using very realistic design options.

Hence, as presented in [14], alternative design options have been pursued, with the low-temperature co-fired ceramic technology (LTCC) presenting, in our view, the best options for the manufacturing of the ITER HF magnetic sensor. Different designs for 1D and 3D LTCC sensors have been prepared and successfully prototyped in-house, and empirical scaling laws, based on detailed measurements, have been developed for the analyses of their frequency response [15]. We found that by making suitable design choices, the electrical properties of these sensors could be made to meet concurrently all the ITER measurement requirements. Moreover, a very important advantage of the LTCC-type design is its very small occupation required to obtain the same (NA) $)_{\mathrm{EFF}}$, which makes this concept particularly attractive for a not-friendly environment such as ITER. Finally, the LTCC technology is also being considered for the design of the low-frequency magnetic sensors to be used in ITER for equilibrium reconstruction [16].

\section{Acknowledgments}

This work, supported by the European Communities under the contract of Association between EURATOM and CRPP-EPFL, was partly carried out within the framework of the European Fusion Development Agreement. This work was also partly supported by the Swiss National Science Foundation. The views and opinions expressed herein do not necessarily reflect those of the European Commission.

\section{References}

[1] (a) ITER Design Description Document DDD 5.5.A, document reference “N55DDD101-06-12W0.3; ITER Diagnostics Review Meeting, vol. 9-13, 2007, July;

(b) G. Vayakis, et al., Rev. Sci. Instrum. 74 (2003) 2411.

[2] (a) E.J. Strait, et al., Fusion Sci. Technol. 53 (2) (2008) 304;

(b) A.J.H. Donné, et al., "Chapter 7: Diagnostics", Special Issue of Nucl. Fusion 47 (2007).

[3] (a) G. Vlad, et al., Plasma Phys. Controlled Fusion 46 (2006) 1; (b) S. Pinches, et al., Nucl. Fusion 46 (2006) S904;

(c) M.P. Gryaznevich, S.E. Sharapov, Nucl. Fusion 46 (2006) S942.

[4] R. Heeter, et al., Rev. Sci. Instrum. 71 (11) (2000) 4092.

[5] S. Guenter, et al., Nucl. Fusion 47 (2007) 920.

[6] M.J. Hole, L.C. Appel, Plasma Phys. Controlled Fusion 51 (2009) 045002.

[7] E.J. Strait, Rev. Sci. Instrum. 77 (2006) 023502, and references therein.

[8] K. Shinohara, et al., Plasma Phys. Controlled Fusion 46 (2004) S31, and references therein.

[9] ITPA Working Group 4 on MHD control.

10] S. Bourguignon, H. Carfantan, T. Böhm, Astron. Astrophys. 462 (2007) 379.

[11] D. Testa, et al., Fusion Sci. Technol. 57 (3) (2010) 208-273.

[12] ITER Project, Risk Management Plan: documents ITER_D_22F4LE (v1.1), 2EW3E7 (v1.2)

[13] D. Testa, et al., Fusion Sci. Technol., submitted for publication.

[14] M. Toussaint. SOFT 2010 Conference proceedings.

[15] D. Testa, et al., Fusion Science and Technology, Fusion Sci. Technol. 59 (2) (2011) 376.

[16] A. Gallo, et al., SOFT 2010 Conference proceedings. 